PROCEEDINGS OF THE

AMERICAN MATHEMATICAL SOCIETY

Volume 129, Number 5, Pages 1459-1462

S 0002-9939(00)05687-2

Article electronically published on October 24, 2000

\title{
STRICTLY POSITIVE DEFINITE FUNCTIONS ON A COMPACT GROUP
}

\author{
MOHAMED ALLALI AND TOMASZ PRZEBINDA \\ (Communicated by Roe Goodman)
}

\begin{abstract}
We recognize a result of Schreiner, concerning strictly positive definite functions on a sphere in an Euclidean space, as a generalization of Bochner's theorem for compact groups.
\end{abstract}

The purpose of this note is to provide a rapid proof of a relatively new result of Schreiner, $[\underline{\mathrm{S}}$, concerning strictly positive definite functions on a sphere in a Euclidean space. It is our pleasure to see that the theory of such functions has found applications in geosciences, and that it is well rooted in the existing mathematical literature.

Let $G$ be a compact group, and let $H \subseteq G$ be a closed subgroup, such that the quotient $G / H$ is infinite. Let $f: G \rightarrow \mathbb{C}$ be a continuous function, invariant under the left and right translations by elements of $H$. We denote the space of all such functions by $C(H \backslash G / H)$. Following Schreiner, [S], we say that the function $f$ is strictly positive definite if and only if

$$
\sum_{i, j=1}^{n} c_{i} \bar{c}_{j} f\left(x_{i}^{-1} x_{j}\right)>0
$$

for any finite set $\left\{x_{1}, x_{2}, \ldots, x_{n}\right\} \subseteq G$ such that the cosets $x_{1} H, x_{2} H, \ldots, x_{n} H \subseteq G / H$ are distinct, and any complex numbers $c_{1}, c_{2}, \ldots, c_{n}$, not all equal to zero.

Let $\hat{G}$ denote the unitary dual of $G,[\underline{K}, 7.3]$. This is the set of equivalence classes of irreducible unitary representations of $G$. For convenience, we choose an irreducible representation for each such class, and identify $\hat{G}$ with the set of these representations. Recall the Fourier transform

$$
\hat{f}(\pi)=\pi(f)=\int_{G} \pi(x) f(x) d x \quad(\pi \in \hat{G}),
$$

where $f$ is any absolutely integrable function on $G$ with respect to the Haar measure $d x$. Thus each $\pi(f)$ is a linear map on the finite dimensional Hilbert space $\mathcal{H}_{\pi}$, where the representation $\pi$ is realized. Let $\mathcal{H}_{\pi}^{H}=\left\{v \in \mathcal{H}_{\pi}: \pi(h) v=v, h \in H\right\}$ be the subspace of $H$-fixed vectors. Let $(G / H)^{\wedge}=\left\{\pi \in \hat{G} ; \mathcal{H}_{\pi}^{H} \neq 0\right\}$. By the Frobenius reciprocity theorem, $(G / H)^{\wedge}$ is the subset of $\hat{G}$, consisting of representations which occur in $L^{2}(G / H)$ (see [K, 8.4]).

Received by the editors December 18, 1998 and, in revised form, August 30, 1999.

1991 Mathematics Subject Classification. Primary 43A35, 43A90, 42A82, 41A05.

Key words and phrases. Positive definite functions, Bochner's theorem.

This research was partially supported by NSF Grant DMS 9622610. 
Proposition 3. A function $f \in C(H \backslash G / H)$ is strictly positive definite if $\pi(f) \geq 0$ for all $\pi \in(G / H)^{\wedge}$, and $\left.\pi(f)\right|_{\mathcal{H}_{\pi}^{H}}>0$ for all but finitely many $\pi \in(G / H)^{\wedge}$.

Here " $\pi(f) \geq 0$ " means that the operator $\pi(f)$ is positive semi-definite, i.e. $(\pi(f) v, v) \geq 0$, for any $v \in \mathcal{H}_{\pi}$, and the statement " $\left.\pi(f)\right|_{\mathcal{H}_{\pi}^{H}}>0$ " means that the restriction of the operator $\pi(f)$ to $\mathcal{H}_{\pi}^{H}$ is positive definite, i.e. $(\pi(f) v, v)>0$, for any $v \in \mathcal{H}_{\pi}^{H} \backslash\{0\}$.

Proof. With the notation (1), let $\mu$ be a bounded measure on $G$ defined by

$$
\int_{G} \phi(x) d \mu(x)=\sum_{i=1}^{n} c_{i} \int_{H} \phi\left(x_{i} h\right) d h
$$

where $\phi$ is a continuous function on $G$, and $d h$ is the Haar measure on $H$, normalized so that the total measure of $H$ is 1 . Notice that the support of $\mu$ is contained in $\bigcup_{i=1}^{n} x_{i} H$, which (by our assumption) is a proper subset of $G$.

The Fourier transform of $\mu$ is defined as in (2):

$$
\pi(\mu)=\int_{G} \pi(x) d \mu(x) \quad(\pi \in \hat{G}) .
$$

Recall the Fourier inversion formula ([K, 12.2] or [H-R, 27.40]):

$$
\phi(x)=\sum_{\pi \in \hat{G}} d(\pi) \operatorname{tr}\left(\pi(\phi) \pi(x)^{*}\right) \quad(\phi \in C(G), x \in G),
$$

where $d(\pi)=\operatorname{dim} \mathcal{H}_{\pi}$. If each operator $\pi(\phi)$ is positive semi-definite $(\pi(\phi) \geq 0)$, then the above Fourier series is absolutely, and hence uniformly, convergent to the continuous function $\phi$ (see [H-R (34.9]). Hence,

$$
\begin{aligned}
f\left(x_{i}^{-1} x_{j}\right) & =\sum_{\pi \in \hat{G}} d(\pi) \operatorname{tr}\left(\pi(f) \pi\left(x_{i}^{-1} x_{j}\right)^{*}\right) \\
& =\sum_{\pi \in \hat{G}} d(\pi) \operatorname{tr}\left(\pi\left(x_{i}\right) \pi(f) \pi\left(x_{j}\right)^{*}\right) .
\end{aligned}
$$

Notice that $\pi(h) \pi(f)=\pi(f)=\pi(f) \pi(h)$ for all $h \in H$. Hence $\pi(f)=P_{\mathcal{H}_{\pi}^{H}} \pi(f)=$ $\pi(f) P_{\mathcal{H}_{\pi}^{H}}$, where $P_{\mathcal{H}_{\pi}^{H}}=\int_{H} \pi(h) d h$ is the orthogonal projection on $\mathcal{H}_{\pi}^{H}$. Therefore,

$$
f\left(x_{i}^{-1} x_{j}\right)=\sum_{\pi \in(G / H)^{\wedge}} d(\pi) \operatorname{tr}\left(\pi\left(x_{i}\right) P_{\mathcal{H}_{\pi}^{H}} \pi(f)\left(\pi\left(x_{j}\right) P_{\mathcal{H}_{\pi}^{H}}\right)^{*}\right) .
$$

After multiplying both sides of (4) by $c_{i} \bar{c}_{j}$ and summing over the indices $i, j$, we see that

$$
\sum_{i, j=1}^{n} c_{i} \bar{c}_{j} f\left(x_{i}^{-1} x_{j}\right)=\sum_{\pi \in(G / H)^{\wedge}} d(\pi) \operatorname{tr}\left(\pi(\mu) P_{\mathcal{H}_{\pi}^{H}} \pi(f)\left(\pi(\mu) P_{\mathcal{H}_{\pi}^{H}}\right)^{*}\right) .
$$

Since $\pi(\mu)=\pi(\mu) P_{\mathcal{H}_{\pi}^{H}}$, the proposition shall follow as soon as we check that

$$
\pi(\mu) \neq 0 \text { for infinitely many } \pi \in(G / H)^{\wedge} \text {. }
$$

Suppose $\mu(\pi)=0$ for all but finitely many $\pi \in(G / H)^{\wedge}$. Then, by Fourier inversion, [H-R, 27.40], $\mu$ coincides with a finite linear combination of matrix coefficients of irreducible unitary representations of $G$. Therefore, the space of all left translates of $\mu$ is finite dimensional. Hence, there are finitely many elements $y_{1}, y_{2}, \ldots, y_{m} \in$ $G$, such that $\bigcup_{j=1}^{m} \operatorname{supp} \mu=G$, where supp $\mu$ stands for the support of $\mu$. Thus $G=\bigcup_{j=1}^{m} \bigcup_{i=1}^{n} y_{j} x_{i} H$, contrary to our assumption that the set $G / H$ is infinite. 
Suppose that $(G, H)$ is a Gelfand pair, [D, 22.6.2]. Then for each $\pi \in \hat{G}$ and each $f \in C(H \backslash G / H)$, the operator $\pi(f)$ is a constant multiple of the projection $P_{\mathcal{H}_{\pi}^{H}}: \pi(f)=\lambda_{\pi}(f) P_{\mathcal{H}_{\pi}^{H}}$.

Corollary 6. If $(G, H)$ is a Gelfand pair, then a function $f \in C(H \backslash G / H)$ is strictly positive definite if $\lambda_{\pi}(f)>0$ for all but finitely many $\pi \in(G / H)^{\wedge}$.

As is well known, [D, 20.11.4, 22.6.3], $(S O(n), S O(n-1))$ is a Gelfand pair, and the quotient space $S O(n) / S O(n-1)$ coincides with the unit sphere in $\mathbb{R}^{n}$. Furthermore, in this case, the scalars $\lambda_{\pi}(f)$ can be expressed in terms of Legendre polynomials. Hence Corollary 6 generalizes a result of Schreiner, [S, Theorem 5.1]. We explain this in some detail.

Consider the Euclidean space $\mathbb{R}^{n}$ equipped with the usual dot product. Let $e_{n}=(0,0, \ldots, 0,1) \in \mathbb{R}^{n}$ be the "north pole" of the unit sphere $S^{n-1} \subseteq \mathbb{R}^{n}$. Let $G=S O(n)$ be the group of isometries of the dot product, and let $H \subseteq G$ be the stabilizer of $e_{n}$. The restriction to the first $n-1$ coordinates identifies $H$ with $S O(n-1)$.

It is customary, in representation theory, to normalize the Haar measure on any compact group to have the total mass 1 . Then the total mass of our homogeneous space $G / H$ is also 1 . On the other hand the dot product in $\mathbb{R}^{n}$ forces a normalization of the rotation invariant measure $\omega$ on the sphere $S^{n-1}$ so that the area of the sphere $\left|S^{n-1}\right|=\int_{S^{n-1}} d \omega \neq 1$. Hence,

$$
\int_{S^{n-1}} \phi(\sigma) d \omega(\sigma)=\left|S^{n-1}\right| \int_{G} \phi\left(g e_{n}\right) d g \quad\left(\phi \in C\left(S^{n-1}\right)\right) .
$$

Therefore the map

$$
L^{2}(G / H) \ni v \rightarrow \phi \in L^{2}\left(S^{n-1}\right), \quad \phi\left(g e_{n}\right)=\left|S^{n-1}\right|^{1 / 2} v(g) \quad(g \in G)
$$

is an isometry. This isometry is surjective and commutes with the action of the group $G$ on both spaces. As is well known, [M], the space $L^{2}\left(S^{n-1}\right)$ decomposes into a direct sum of irreducible subspaces:

$$
L^{2}\left(S^{n-1}\right)=\sum_{m=0}^{\infty} L^{2}\left(S^{n-1}\right)_{m}
$$

where $L^{2}\left(S^{n-1}\right)_{m}$ stands for the space of spherical harmonics of degree $m$. Let $\pi_{m}$ denote the representation of $G$ on the space $\mathcal{H}_{\pi_{m}}=L^{2}\left(S^{n-1}\right)_{m}$. Then the subspace $\mathcal{H}_{\pi_{m}}^{H}$ is one dimensional, and is spanned by the Legendre function:

$$
L_{m}(\sigma)=P_{m}\left(e_{n} \cdot \sigma\right) \quad\left(\sigma \in S^{n-1}\right)
$$

where $P_{m}$ is the Legendre polynomial of degree $m$ (see [M, Lemma 2, page 16]). Notice that a function $f \in C(H \backslash G / H)$ is uniquely determined by a continuous function $F$ on the interval $[-1,1]$ by the formula

$$
f(g)=F\left(e_{n} \cdot g e_{n}\right) \quad(g \in G) .
$$

Notice that $f(g)=F\left(e_{n} \cdot g e_{n}\right)=F\left(g^{-1} e_{n} \cdot e_{n}\right)=F\left(e_{n} \cdot g^{-1} e_{n}\right)=f\left(g^{-1}\right)$. In these terms, the constants which occur in Corollary 6 can be calculated as follows (see 
[M] Lemma 1 on page 15 , and formula (§4.6) on page 29]):

$$
\begin{aligned}
\lambda_{\pi_{m}}(f) & =\lambda_{\pi_{m}}(f) L_{m}\left(e_{n}\right)=\pi_{m}(f) L_{m}\left(e_{n}\right) \\
& =\int_{G} f(g) L_{m}\left(g^{-1} e_{n}\right) d g=\int_{G} F\left(e_{n} \cdot g e_{n}\right) P_{m}\left(e_{n} \cdot g^{-1} e_{n}\right) d g \\
& =\int_{G} F\left(e_{n} \cdot g e_{n}\right) P_{m}\left(e_{n} \cdot g e_{n}\right) d g=\frac{\left|S^{n-2}\right|}{\left|S^{n-1}\right|} \int_{-1}^{1} F(t) P_{m}(t)\left(1-t^{2}\right)^{(n-3) / 2} d t .
\end{aligned}
$$

Thus, by Corollary 6 , the integral kernel operator on $L^{2}\left(S^{n-1}\right)$ corresponding to the integral kernel $F(\eta, \sigma)=F(\eta \cdot \sigma),\left(\eta, \sigma \in S^{n-1}\right)$ is strictly positive definite if $\lambda_{\pi_{m}} \geq 0$ for all $m \geq 0$, and the inequality is strict for all but finitely many $m$. This is the content of Theorem 5.1 of Schreiner, $[\mathbf{S}$.

\section{ACKNowledgement}

We would like to thank the referee for a very careful revision of this work.

\section{REFERENCES}

[D] J. Dieudonné, Éléments d'Analyse, Gauthier-Villars Éditeur, Paris/Bruxelles/Montréal, 1975. MR 58:29825a

[H-R] E. Hewitt and K. A. Ross, Abstract Harmonic Analysis, Volume II, Springer-Verlag, New York, 1970. MR 41:7378

[K] A. A. Kirillov, Elements of the Theory of Representations, Springer-Verlag, New York, 1976. MR 54:447

[M] C. Müller, Analysis of Spherical Symmetries in Euclidean Spaces, Springer-Verlag, New York, 1998. CMP 98:05

[S] M. Schreiner, On a New Condition For Strictly Positive Definite Functions On Spheres, Proceedings of the AMS 125 (1997), 531-539. MR 97d:43005

Department of Mathematics, University of Oklahoma, Norman, Oklahoma 73019

E-mail address: mallali@crystal.math.ou.edu

Current address: Department of Computer Science, Mathematics and Physics, Chapman University, Orange, California 92866

Department of Mathematics, University of Oklahoma, Norman, Oklahoma 73019 